\title{
Static and Dynamic Analysis of Bistable Piezoelectric- Composite Plates for Energy Harvesting
}

\author{
David N. Betts ${ }^{1}$, H. Alicia Kim ${ }^{2}$, and Christopher R. Bowen ${ }^{3}$ \\ Department of Mechanical Engineering, University of Bath, Bath, BA2 7AY, United Kingdom \\ and \\ Daniel J. Inman ${ }^{4}$ \\ Department of Aerospace Engineering, University of Michigan, Ann Arbor, MI, 48109, USA
}

\begin{abstract}
This paper presents an arrangement of bistable composite plates with bonded piezoelectric patches to perform broadband vibration-based energy harvesting from ambient mechanical vibrations. These bistable nonlinear devices have the potential to exhibit improved power generation compared to conventional resonant systems and can be designed to occupy smaller volumes than bistable magnetic cantilever systems. In this paper we initially present the results of an optimization study to generate greater electrical power by discovering the correct geometric configuration for energy harvesting based on the static states of the device. The results consider the optimal choice of device aspect ratio, laminate thickness, laminate stacking sequence, and piezoelectric surface area. Increased electrical output is found for geometries and piezoelectric configurations which have not been considered previously. This study is then extended to include dynamic considerations of both the static shapes and the snap-through transition. Optimum designs are shown to be sensitive to the vibration pattern that is being harvested. The optimum geometric configurations based on the static analysis alone are not optimal under all dynamic conditions.
\end{abstract}

\section{Introduction}

$\mathrm{E}$ NERGY harvesting which converts ambient mechanical vibrations into electrical energy is an area of considerable research interest and has received extensive attention in the past decade ${ }^{1}$. A variety of methods have been considered including electrostatic generation ${ }^{2}$, electromagnetic induction ${ }^{3}$ and the piezoelectric effect ${ }^{4}$. Priya $^{5}$ demonstrated that piezoelectrics have a number of advantages, including ease of integration within a system, higher strain energy densities compared to electrostatic and electromagnetic systems, and the simplicity of converting strain energy to electrical energy. In many cases harvesting devices have been designed to operate near resonance to optimize the power generation, for example simple linear cantilever beam configurations. However, the ambient vibrations which are intended to be harvested generally exhibit multiple time-dependent frequencies which can include components at relatively low frequencies. This can make typical linear systems inefficient or unsuitable; particularly if the resonant frequency of the device is much higher than the frequency range of the vibrations it is attempting to harvest ${ }^{6}$. In order to improve the efficiency of vibrational energy harvesters, recent work has focused on exploiting nonlinearity for broadband energy harvesting. Encouraging results ${ }^{7-8}$ have been obtained using nonlinear or bistable cantilevered beams. Stanton et al. ${ }^{8}$ modeled and experimentally validated a nonlinear energy harvester using a piezoelectric cantilever. An end magnet was placed on an oscillating cantilever which then interacts with oppositely poled, stationary magnets which induces softening or hardening into the system and allows the resonance frequency to be tuned. This technique was shown to outperform linear systems when excited by varying frequencies. However, such a system would require an obtrusive arrangement of external magnets and could generate unwanted electromagnetic fields. An alternative method ${ }^{4}$ has recently been presented where a piezoelectric element is attached to the surface of an asymmetric and bistable laminate plate, exploiting the large structural

\footnotetext{
${ }^{1}$ Research Officer, Department of Mechanical Engineering, D.N.Betts@bath.ac.uk.

${ }^{2}$ Senior Lecturer, Department of Mechanical Engineering, H.A.Kim@bath.ac.uk.

${ }^{3}$ Professor, Department of Mechanical Engineering, C.R.Bowen@ bath.ac.uk.

${ }^{4}$ Professor, Department of Aerospace Engineering, daninman@umich.edu.
} 
change which is enabled by the inherent bistability. Such harvesting structures have been shown to exhibit high levels of power extraction over a wide range of frequencies. This arrangement can be designed to occupy a smaller space and is potentially more convenient and portable for broadband energy harvesting.

Bistable laminates have been extensively studied for the development of morphing or adaptive structure concepts $^{9-12}$. When a composite laminate has an asymmetric stacking sequence the resulting mismatch in thermal properties between plies leads to a thermally induced strain. This leads to the laminate developing a curved deformation as it is cooled from its high temperature cure cycle to room temperature. Under certain geometric conditions the thermal strain can lead to the development of two stable equilibrium states. Such structures are of interest for shape-change applications since the 'snap-through' between stable states can result in a large deflection but does not require continuous energy input to maintain the stable shape. Figure 1 shows an example of this behavior for a square $\left[0_{\mathrm{n}} / 90_{\mathrm{n}}\right]_{\mathrm{T}}$ laminate. For a low ratio of edge length to thickness only a saddle shaped single stable state is observed, point $\mathrm{A}$, with $x$ - and $y$-curvatures of equal magnitude in opposite out-of-plane directions. As the ratio increases the solution bifurcates, point $\mathrm{B}$. Beyond this point two approximately cylindrical stable states are observed, points $\mathrm{C}$ and $\mathrm{D}$, while the saddle state becomes unstable (dashed line). For morphing applications piezoelectric materials can be used to induce 'snap-through' between points $\mathrm{C}$ and $\mathrm{D}$. For harvesting applications, if a flexible piezoelectric material is attached to the laminate surface and the structure is repeatedly actuated between stable states $\mathrm{C}$ and $\mathrm{D}$ as a result of a mechanical vibration, the large shape change has the potential to generate electrical energy by the direct piezoelectric effect.

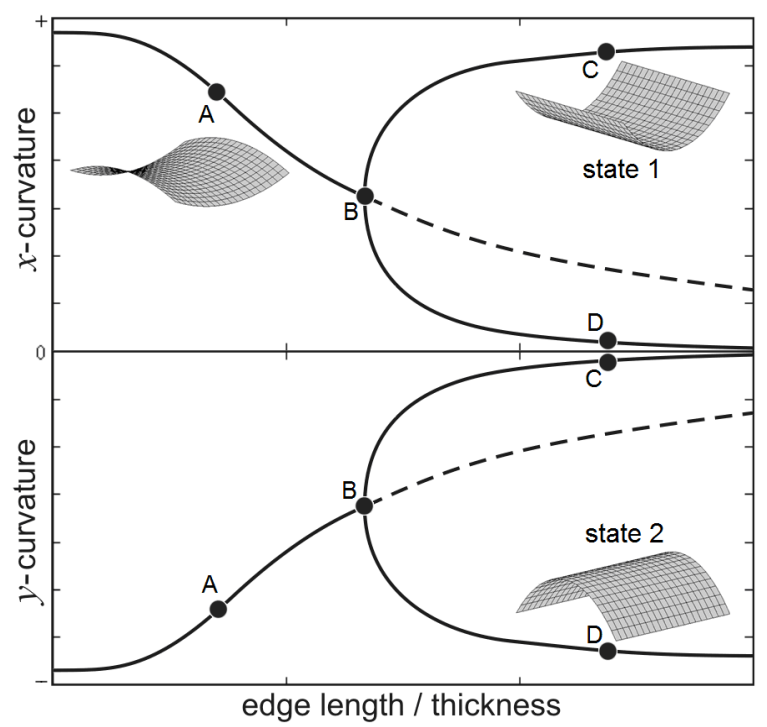

Figure 1. Stable (solid line) and unstable (dashed) shapes of a $\left[0_{n} / 90_{n}\right]_{T}$ laminate with variation in geometry.

Betts et al. ${ }^{13}$ recently presented a study for optimization of the design of bistable laminates for morphing applications, enabled by the derivation of an analytical solution to existing numerical modeling ${ }^{10}$. Through variation of ply orientations and using a nonuniform laminate geometry, laminates were optimized for differing stiffness characteristics, constrained by bistability and minimum deflection constraints. This work was extended ${ }^{14}$ to include piezoelectric layers into the existing analytical model in a configuration similar to that considered experimentally by Arrieta et al. ${ }^{4}$. Most recently this optimization methodology ${ }^{14}$ has been adapted for the design of bistable piezoelectric-laminate energy harvesters based on the static shapes of the device only ${ }^{15}$.

This paper will begin by presenting a summary of the correct geometric configurations of bistable laminate energy harvesters based purely on static considerations. The variables considered are the stacking sequence of the composite laminate, ply thicknesses, aspect ratio and piezoelectric configurations. These results are further discussed with a series of design parameter studies to provide a better understanding of the optimum results. Modeling methods are then extended to include dynamic considerations of the vibrational input and the time dependency of the laminate during snap-through transitions. With consideration of the amplitude of available actuation force, optimal geometric configurations are shown to be sensitive to the vibration pattern which is being harvested. 


\section{Device Configuration}

An existing experimental study presented by Arrieta et al. ${ }^{4}$ suggested a novel mechanism for broadband energy harvesting of ambient mechanical vibrations, using composite laminates with an asymmetric layup. When subjected to mechanical vibrations these bistable laminates can exhibit large amplitude oscillations. Arrieta et al. ${ }^{4}$ have previously demonstrated that this alternating stress excitation can induce high levels of electrical energy in bonded piezoelectric layers across a wide range of frequencies. Figure 2 shows the experimental arrangement considered ${ }^{4}$, using a $\left[0_{2} / 90_{2}\right]_{\mathrm{T}}$ laminate of $0.2 \mathrm{~m}$ square edge-length with four bonded piezoelectric layers for energy harvesting. The bistable piezoelectric-laminate combination was attached to a shaker and seismic masses attached to the corners of the laminate to facilitate snap-through between stable states.

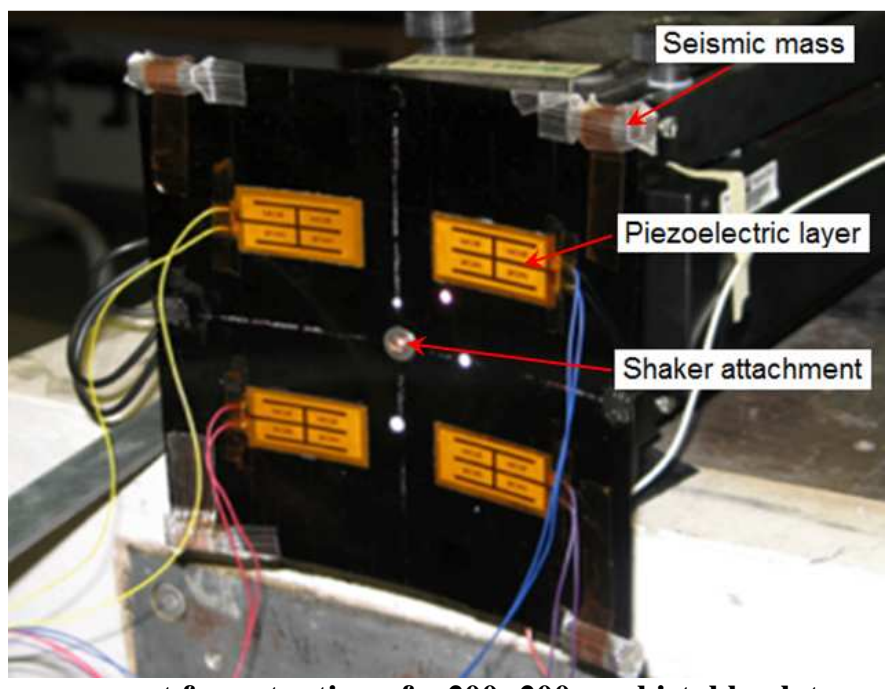

Figure 2. Experimental arrangement for actuation of a $200 \times 200 \mathrm{~mm}$ bistable plate mounted from its center to a mechanical shaker. Adapted from Arrieta et al. ${ }^{4}$.

While this type of device was shown to produce large average power from intermittent, limit cycle and chaotic oscillations, the design of the device was not optimized to maximize energy outputs. In this work we look to exploit the many variables available in the design of such a piezoelectric-laminate structure to improve harvesting performance. An example arrangement considered in this work is shown in Fig. 3, with a similar pattern of four piezoelectric layers positioned on the top laminate surface and mirrored on the opposite surface by a further four piezoelectric layers positioned orthogonally to those on the upper surface.

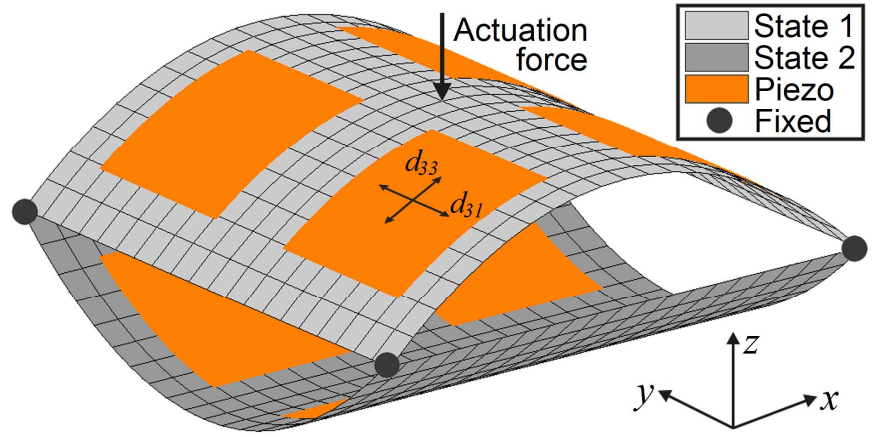

Figure 3. Actuation arrangement for a $\left[0^{\mathrm{P}} / \mathbf{0}_{2} / 90_{2} / 90^{\mathrm{P}}\right]_{\mathrm{T}}$ laminate with $40 \%$ piezoelectric coverage. Superscript $P$ denotes piezoelectric element with $0^{\circ}$ or $90^{\circ}$ poling direction.

To approximate the effect of ambient mechanical vibrations the device is modeled as fixed at the four corners (zero $z$-displacement) with a mechanical force applied in the $z$-direction at the geometric center. The design variables which have been considered in this study are the stacking sequence of the cross-symmetric laminate, the planform dimensions of the rectangular device, ply thicknesses, and total piezoelectric area. 


\section{Static Analysis}

In this section the optimal geometric configurations of the piezoelectric-laminate energy harvesters are considered based solely on the static shapes of the system. Piezoelectric elements are assumed to behave as parallel plate capacitors, constrained by a maximum strain to avoid material failure, but with no consideration for amplitude or frequency of the source of the mechanical actuation.

\section{A. Model for Static Laminate Shapes}

The analytical model used to calculate the unloaded shapes of asymmetric laminates of arbitrary layup was first introduced by Dano and Hyer ${ }^{10}$ and is a nonlinear extension to classical laminated plate theory. The co-ordinate system used is that defined in Fig. 3, where the geometric centre of the laminate sits at the origin and the ply orientations are measured from the $x$-axis. The out-of-plane displacement in the $z$-direction, $w$ is assumed to be of the following form.

$$
w=\frac{1}{2}\left(a x^{2}+b y^{2}+c x y\right)
$$

The midplane strains, including geometrical nonlinearity according to the von Karman hypothesis, are defined as

$$
\begin{aligned}
& \varepsilon_{x}^{0}=\frac{\partial u^{0}}{\partial x}+\frac{1}{2}\left(\frac{\partial w}{\partial x}\right)^{2} \\
& \varepsilon_{y}^{0}=\frac{\partial v^{0}}{\partial y}+\frac{1}{2}\left(\frac{\partial w}{\partial y}\right)^{2} \\
& \varepsilon_{x y}^{0}=\frac{\partial u^{0}}{\partial y}+\frac{\partial v^{0}}{\partial x}+\frac{1}{2} \frac{\partial w}{\partial x} \frac{\partial w}{\partial y}
\end{aligned}
$$

where $u^{0}$ and $v^{0}$ are the in-plane displacements in the $x$ - and $y$-directions, respectively. The midplane strains are approximated by third order polynomials. Dano and Hyer $^{10}$ found that terms with powers of $x$ and $y$ that sum to an odd number are always zero. Therefore the the midplane strains can be reduced to the polynomials of Eq. (3).

$$
\begin{aligned}
& \varepsilon_{x}^{0}=e_{1}+e_{2} x^{2}+e_{3} x y+e_{4} y^{2} \\
& \varepsilon_{y}^{0}=e_{5}+e_{6} x^{2}+e_{7} x y+e_{8} y^{2}
\end{aligned}
$$

Using Eqs. (1-3) and introducing the additional shape coefficients $e_{9-11}$ resulting from integration of the midplane strains, expressions for the in-plane displacements $u^{0}$ and $v^{0}$ can be determined.

$$
\begin{aligned}
& u^{0}(x, y)=e_{1} x+e_{9} y+\frac{1}{2}\left(e_{3}-\frac{1}{2} a c\right) x^{2} y+\left(e_{4}-\frac{c^{2}}{8}\right) x y^{2}+\frac{1}{3}\left(e_{2}-\frac{1}{2} a^{2}\right) x^{3}+\frac{1}{3} e_{11} y^{3} \\
& v^{0}(x, y)=e_{9} x+e_{5} y+\frac{1}{2}\left(e_{7}-\frac{1}{2} b c\right) x y^{2}+\left(e_{6}-\frac{c^{2}}{8}\right) x^{2} y+\frac{1}{3}\left(e_{8}-\frac{1}{2} b^{2}\right) y^{3}+\frac{1}{3} e_{10} x^{3}
\end{aligned}
$$

The total strain energy of the laminate, $W_{\text {lam }}$ can then be expressed as the integral of strain energy density over the volume of the laminate.

$$
W_{l a m}=\int_{-L_{x} / 2}^{L_{x} / 2} \int_{-L_{y} / 2}^{L_{y} / 2} \int_{-H / 2}^{H / 2} \frac{1}{2} c_{i j k l} \varepsilon_{i j} \varepsilon_{k l}-\hat{\alpha}_{i j} \varepsilon_{i j} \Delta T d x d y d z
$$

where $c_{i j k l}$ 's are elastic constants, $\hat{\alpha}_{i j}$ 's are constants relating to the thermal expansion coefficients, $L_{x}$ and $L_{y}$ are the planform side lengths of the laminate, $H$ is the total laminate thickness, $\Delta T$ is the temperature change from the cure temperature and $\varepsilon_{i j}$ 's and $\varepsilon_{k l}$ 's are the total strains defined as, 


$$
\begin{aligned}
& \varepsilon_{x}=\varepsilon_{x}^{0}-z a \\
& \varepsilon_{y}=\varepsilon_{y}^{0}-z b \\
& \varepsilon_{x y}=\varepsilon_{x y}^{0}-z c
\end{aligned}
$$

where $z$ is the out-of-plane distance from the laminate midplane and $a, b$, and $c$ are out-of-plane shape coefficients defined by Eq. (1). To include the additional stiffness of the piezoelectric layers an additional strain energy term $W_{p}$ is added, dependent on the geometry and material properties of the individual layers. The piezoelectric elements are typically bonded to the laminate at room temperature after the cure cycle and to account for this the temperature change is set to zero. The total strain energy of the piezoelectric-laminate device $W$ is then the sum of the laminate and piezoelectric components,

$$
W=W_{l a m}+W_{p}
$$

Expansion of Eq. (7) results in an expression for the total strain energy which is a function of the material and geometric properties, the temperature change from cure and the set of shape coefficients $a, b, c, e_{1} \ldots e_{11}$. For equilibrium, the minimum energy states require:

$$
f_{i}=\frac{\partial W}{\partial k_{i}}=0 ; \quad i=1 \ldots 14
$$

where the $k_{i}$ 's are the set of 14 shape coefficients. In this form it is noted that throughout the 14 equations the shape coefficients $e_{1-11}$ appear independently and as linear terms only. However the equations are not linear with respect to the out-of-plane coefficients $a, b$ and $c$. The system can therefore be reduced to just three equations and the three unknown curvatures $a, b$ and $c$. This process results in a system defining the equilibrium positions of the following form, which can be solved efficiently using numerical solution methods.

$$
\frac{\partial W(a, b, c)}{\partial a}=\frac{\partial W(a, b, c)}{\partial b}=\frac{\partial W(a, b, c)}{\partial c}=0
$$

\section{B. Objective Function}

The objective of this study is to maximize the electrical energy generated by the bistable piezoelectric-laminate structure during snap-through from each stable state. At this stage we model this based purely on the static stable states of the system. The objective function is thus the electrical energy, $U$ induced in the piezoelectric material under an applied stress which results in a transition between the two stable states determined in the previous section. When operating off-resonance a piezoelectric layer behaves as a parallel plate capacitor. Hence the electrical energy generated by snap-through is given by,

$$
U=\frac{1}{2} C V^{2}
$$

where $C$ is the capacitance of the piezoelectric element and $V$ is the open circuit output voltage, as defined by the following equations,

$$
\begin{gathered}
C=\frac{Q}{V}=\frac{d_{i j} \sigma A}{V} \\
V=E t=-g_{i j} \sigma t_{p}
\end{gathered}
$$

where $Q$ is charge, $d_{i j}$ is the effective piezoelectric strain constant (charge per unit force), $g_{i j}$ is the effective piezoelectric voltage constant (electric field per unit stress), $\sigma$ is the stress, $A$ is the surface area of the layer, and $t_{p}$ is the thickness of the layer. Substituting Eqs. (11) and (12) into (10) gives, 


$$
U=\frac{1}{2}\left(d_{i j} g_{i j}\right) \cdot \sigma^{2} \cdot\left(A t_{p}\right)
$$

where the terms have been split into the fixed material properties of the piezoelectric material $\left(d_{i j}\right.$ and $\left.g_{i j}\right)$, the stress in the material which is a function of the laminate curvature, and the material geometry $\left(A\right.$ and $\left.t_{p}\right)$. In this analysis it is assumed that the piezoelecitrc element is polarized along fiber axis of the laminate (as in Fig 3). This is achieved using a Macro Fiber Composite piezoelectric device with an inter-digitated electrode pattern to align the electric field along the fiber axis. When attached to the laminate surface the piezo-elements are strained in both the poling direction (33 direction, Fig. 3) and transverse direction (31 direction) due to the anticlastic curvatures ${ }^{9}$. The stress varies across the volume of the piezoelectric-elements as a function of the thermally induced stresses. The electrical energy for piezoelectric-elements positioned at $0^{\circ}$ (top surface) and $90^{\circ}$ (bottom surface) is therefore,

$$
U=4 \sum_{m=1}^{2}\left[\frac{1}{2} \int_{v_{1}}\left(d_{33} g_{33} \sigma_{x}^{2}+d_{31} g_{31} \sigma_{y}^{2}\right) d v_{1}+\frac{1}{2} \int_{v_{2}}\left(d_{33} g_{33} \sigma_{y}^{2}+d_{31} g_{31} \sigma_{x}^{2}\right) d v_{2}\right]
$$

where the factor 4 accounts for all piezoelectric-elements on one surface, $m$ defines the associated shape, and $v_{1}$ and $v_{2}$ are the volumes of two layers on opposite laminate surfaces. The associated material properties and stress directions for each component are more clearly illustrated in Fig. 4. It is interesting to note that while an increase in piezoelectric-element size increases the area from which electrical energy can be harvested, their additional stiffness reduces laminate curvature and thus the resulting stress $\sigma$. Other potential configuations of the piezoelectric element include polarization in the through-thickness direction ( $z$-axis in Fig. 4 ) so that the $d_{31}$ coefficient is responsible for harvesting in both $x$ and $y$ direction; this is not considered in this paper for brevity.

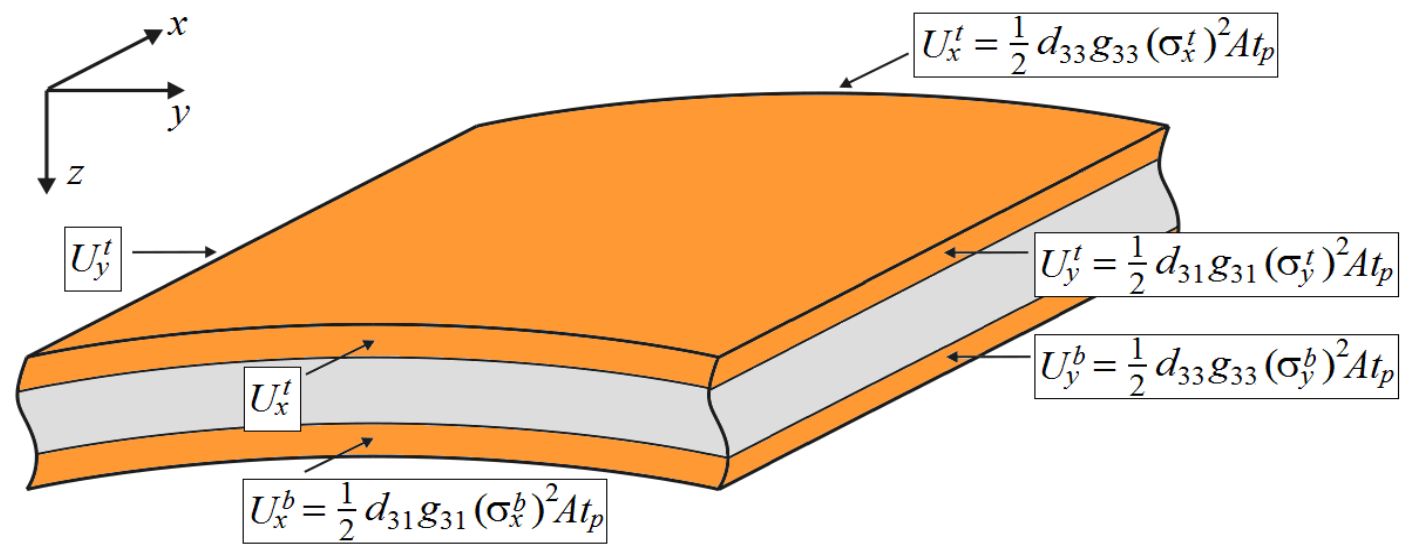

Figure 4. Longitudinal and transverse components of the total electrical energy, $U$. Note: subscript denotes the associated direction, superscript denotes the top (t) and bottom (b) piezoelectric layer.

\section{Results}

For the optimization study the material properties $d_{i j}$ and $g_{i j}$ are considered to be fixed as their optimum values have been determined by Priya ${ }^{14}$. The surface area of the laminate is fixed $\left(0.04 \mathrm{~m}^{2}\right)$ as reported by Arrieta et al. ${ }^{4}$. A lower bound on single ply thickness $t$ is set as $0.125 \mathrm{~mm}$, consistent with typical minimum ply thicknesses. $t_{p}$ is fixed for practical reasons. All other variables are unbounded. The optimum solutions are subject to constraints to guarantee bistability ${ }^{14}$ and limiting piezoelectric strain to below its failure $\operatorname{strain}^{16}(\sim 2000 \mu$ strain). The optimization problem is solved using a sequential quadratic programming method with multiple starting points uniformly distributed throughout th design space to capture all local optima.

Two optima are found for this problem, shown in Table 1, with layups of $\left[0^{\mathrm{P}} / 0 / 90 / 90^{\mathrm{P}}\right]_{\mathrm{T}}$ (global optimum) and $\left[0^{\mathrm{P}} / 90 / 0 / 90^{\mathrm{P}}\right]_{\mathrm{T}}$ (local optimum) where the global optimum outperforms the local solution by $\sim 65 \%$. 
Table 1: Global and local optimum solutions.

\begin{tabular}{|l|c|c|}
\hline & Local optimum solution & Global optimum solution \\
\hline Stacking sequence & {$\left[0^{\mathrm{P}} / 90 / 0 / 90^{\mathrm{P}}\right]_{\mathrm{T}}$} & {$\left[0^{\mathrm{P}} / 0 / 90 / 90^{\mathrm{P}}\right]_{\mathrm{T}}$} \\
\hline Single ply thickness, $\mathrm{mm}$ & 0.619 & 0.626 \\
\hline Piezoelectric area, $\%$ & 42.40 & 72.43 \\
\hline Aspect ratio & 1.0 & 1.0 \\
\hline Maximum strain, $\mu$ strain & 1113 & 1097 \\
\hline Actuation force, $\mathrm{N}$ & 3.39 & 3.41 \\
\hline Electrical energy, $\mathrm{mJ}$ & 20.4 & 33.7 \\
\hline
\end{tabular}

For both solutions in Table 1, the major and minor curvatures are aligned with the polarization direction of the piezoelectric to utilise the $d_{33}$ and $d_{31}$ piezoelectric effect, and they are intuitively the optima. The local solution is less optimal due to the reduced curvature (relative to the global solution) associated with this stacking sequence. What are less obvious are the geometric configurations of piezoelectric area and device thickness. The optimum ply thicknesses are $0.626 \mathrm{~mm}$ and $0.619 \mathrm{~mm}$ for the global and local solutions respectively, and are not on any constraint boundary. Conveniently, this global optimum is almost exactly 5 plies of $0.125 \mathrm{~mm}$ meaning the solution could be redefined as $\left[0^{\mathrm{P}} / 0_{5} / 90_{5} / 90^{\mathrm{P}}\right]_{\mathrm{T}}$. Significant differences in the piezoelectric areas for the global $(72.43 \%)$ and local $(42.40 \%)$ solutions are observed. These results are further investigated in following design parameter studies.

\section{Laminate Stacking Sequence}

The first study considers the change in objective function with laminate stacking sequence. Figure 5a shows the electrical energy generated for differing values of $\theta$ for laminates of $\left[0^{\mathrm{P}} / \theta / \theta+90 / 90^{\mathrm{P}}\right]_{\mathrm{T}}$ layup, $0.2 \mathrm{~m}$ square edge length and $0.25 \mathrm{~mm}$ ply thickness. Optima for each $\theta$ value are marked by black squares, while the global and local optima are marked by a white circle and square respectively. Different piezoelectric orientations are not considered as only the angles relative to ply orientations are of interest, but is something that warrants further investigation.

The maximum electrical energy is observed when $\theta$ is $0^{\circ}$, corresponding to the global solution to the optimization problem (white circle). As $\theta$ increases from $0^{\circ}$ to $45^{\circ}$ the major laminate curvature reduces and the alignment of the piezoelectric polarization direction with laminate curvature becomes less optimal, leading to a decrease in the electrical energy. For $\theta$ values from $45^{\circ}$ to $\sim 60^{\circ}$ the major curvature continues to decrease but the piezoelectric alignment improves, the net effect being a more gradual decrease in energy generated. At $\sim 60^{\circ}$ this pattern switches and the improved alignment of the piezoelectric with curvature becomes dominant compared to the reduced curvature and the electrical energy increases until the local solution at $\theta=90^{\circ}$ (white square).
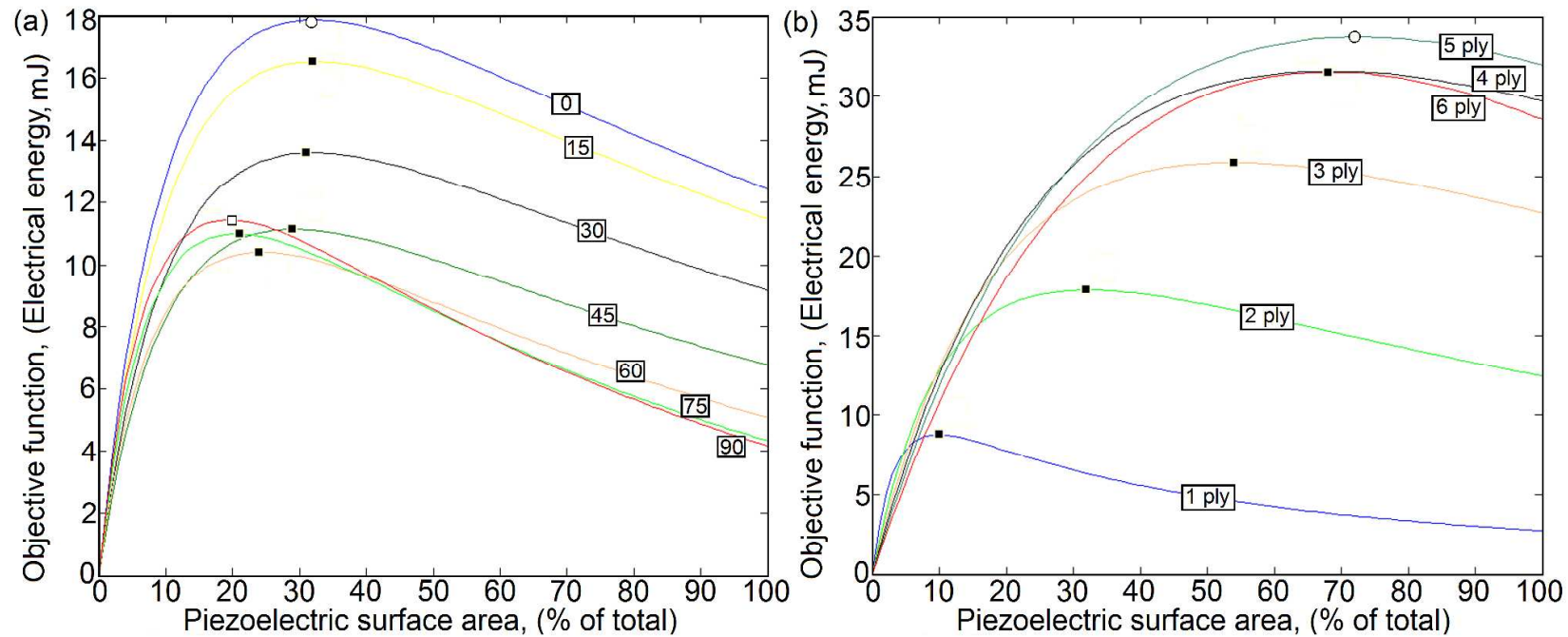

Figure 5. (a) Variation in electrical energy with $\theta$ (shown on each line) and piezoelectric surface area for $\left[0^{\mathrm{P}} / \theta / \theta+90 / 90^{\mathrm{P}}\right]_{\mathrm{T}}$ laminates, and $(\mathrm{b})$ variation in electrical energy with $\mathrm{n} \times 0.125 \mathrm{~mm}$ plies (shown on each line) and piezoelectric area for $\left[0^{\mathrm{P}} / 0_{\mathrm{n}} / 90_{n} / 90^{\mathrm{P}}\right]_{\mathrm{T}}$ laminates. Black squares mark optima for each $\theta$ or $\mathrm{n}$, white circles mark global optima, and white squares mark local optima. 


\section{Device Thickness}

The second study considers the effect of ply thickness on the problem. Figure $5 \mathrm{~b}$ shows the objective function values for differing number of plies ( $\mathrm{n}$ from 1 to 6 ) for the full range of piezoelectric areas. All results correspond to $\left[0^{\mathrm{P}} / 0_{\mathrm{n}} / 90_{\mathrm{n}} / 90^{\mathrm{P}}\right]_{\mathrm{T}}$ laminates of $0.2 \mathrm{~m}$ square edge length. For each different number of plies the optimum solution with respect to piezoelectric area is highlighted by a black square, with the global optimum marked by a white circle.

A consistent pattern of results is observed for ply numbers from 1 to 5 . For zero piezoelectric area the electrical energy generated is obviously zero. The electrical energy then increases with additional piezoelectric area to an optimum value. This optimum area is larger for each additional number of plies up to $72.43 \%$ for 5 plies. However, beyond 5 plies this pattern changes with both the optimum area and the maximum electrical energy decreasing for thicker laminates. This is a result of approaching the bifurcation point (between 6 and 7 plies depending on the piezoelectric surface area), resulting in decreasing curvature until the bistable behavior is lost (see point B in Fig. 1). This pattern confirms the finding that the bistability constraint is inactive at the global optimum.

This result suggests that thicker laminates with lower curvatures are more optimal, which is not an intuitive result. For the thicker design the piezoelectric layers are positioned further from the midplane and thus experience higher strain per unit curvature. However, in this analysis the source and magnitude of the actuation force has not been considered. Clearly, these thicker laminate designs will have higher stiffness and require larger vibrational force input to induce snap-through between the stable states. The optimality of these designs is therefore dependent on the source of vibration to be harvested and will now be investigated.

\section{Dynamic Analysis}

The majority of existing literature for bistable laminate analysis considers the snap-through event to be an instantaneous one, with the dynamics of the transition between the stable states rarely considered. For an application such as energy harvesting where the device performance is dependent on its response to ambient mechanical vibrations, a better understanding of the dynamic transition is vital. In this section the analytical model of Section IIIA is extended using the method outlined by Diaconu et al. ${ }^{17}$, providing a fast and robust method of evaluating the time-dependent respsonse of the bistable piezoelectric-laminate harvesting device.

\section{A. Model for Dynamic Snap-through}

This section presents an overview of the dynamic extension to the analytical model presented by Diaconu et al. ${ }^{17}$. The static stability of the bistable structure subjected to an externally applied force is initially considered. This simple extension to the energy minimization problem of Eq. (9) is performed by solving the following system of three nonlinear equations,

$$
\mathbf{K}(\mathbf{x})-\mathbf{F}(\mathbf{x})=0
$$

where $\mathbf{x}=[a, b, c]^{\mathrm{T}}, \mathbf{K}$ is the vector containing the three unloaded equilibrium equations of Eq. (9), representing a stiffness function,

$$
\mathbf{K}(\mathbf{x})=\left[\frac{\partial W}{\partial a}, \frac{\partial W}{\partial b}, \frac{\partial W}{\partial c}\right]^{\mathrm{T}}
$$

and $\mathbf{F}$ is the force function of $a, b$ and $c$, giving the work done by the applied force,

$$
\mathrm{F}=2 f\left[\left(\frac{L_{x}}{2}\right)^{2},\left(\frac{L_{y}}{2}\right)^{2}, 0\right]^{\mathbf{T}}
$$

where $f$ is the magnitude of the applied force. If $f$ is considered to be constant, solution of Eq. (15) with respect to the curvatures $a, b$ and $c$ will result in the static shapes of the laminate when subjected to the static force. In this case we consider the force $f$ to be varying with time, approximating the alternating mechanical vibrations which are to be captured by the device. For all studies presented in this section, $f$ is assumed to be sinusoidal with a peak amplitude of $f_{\max }$. The dynamic equations of motion are then of the form, 


$$
\mathbf{M} \ddot{\mathbf{x}}+\mathbf{D}(\dot{\mathbf{x}})+\mathbf{K}(\mathbf{x})=\mathbf{F}(\mathbf{x}, t)
$$

where $\mathbf{M}$ is the mass matrix as derived by Diaconu et al. ${ }^{17}$. Here this includes the components relating to only the laminate, $\mathbf{M}_{\text {lam }}$,

$$
\mathbf{M}_{\mathrm{lam}}=\frac{\rho H L_{x} L_{y}}{32}\left[\begin{array}{ccc}
\left(\frac{L_{x}^{4}}{10}-\frac{H^{2} L_{x}^{2}}{9}\right) & \left(\frac{L_{x}^{2} L_{y}^{2}}{18}-\frac{H^{2} L_{x}^{2}}{9}\right) & 0 \\
0 & \left(\frac{L_{x}^{2} L_{y}^{2}}{18}-\frac{H^{2} L_{y}^{2}}{9}-\frac{H^{2} L_{y}^{2}}{9}\right) & 0 \\
0 & \left(\frac{L_{x}^{2} L_{y}^{2}}{18}\right)
\end{array}\right]
$$

where $\rho$ is the laminate material density, and the component relating to the piezoelectric layers $\mathbf{M}_{\mathrm{p}}$ with the corresponding properties for the piezoelectric material. The mass matrix $\mathbf{M}$ of the piezoelectric-laminate combination is simply the sum of $\mathbf{M}_{\mathrm{lam}}$ and $\mathbf{M}_{\mathrm{p}}$. $\mathbf{D}$ is the damping term with a mass damping coefficient $\eta$.

$$
\mathbf{D}(\dot{\mathbf{x}})=\eta \mathbf{M} \dot{\mathbf{x}}
$$

In this work the stiffness damping is omitted as the snap-through event occurs in the low frequency range. Equation (18) can then be solved using Matlab's built in ODE solver ODE15S, using either one of the two static shapes as an initial start point.

\section{B. Results}

As an example of the dynamic response of a bistable piezoelectric-laminate predicted by the model outlined in the previous section, the global optimum design outlined in Section IIIC is initially considered. The harvesting device has a layup of $\left[0^{\mathrm{P}} / 0 / 90 / 90^{\mathrm{P}}\right]_{\mathrm{T}}$, with a single ply thickness of $0.626 \mathrm{~mm}$, a square edge length of $0.2 \mathrm{~m}$, and is excited by an alternating force with a maximum amplitude of $8 \mathrm{~N}$ (see Fig. 6a). The resulting deformation of the piezoelectric-laminate configuration over the first four seconds is shown in Fig. 6b, measured as the displacement of one corner of the device from the flat state. The dashed red lines indicate the two stable shapes as predicted by the static modeling of Section IIIA, and the solid blue line is the motion of the corner displacement of the device with alternating mechanical excitation.
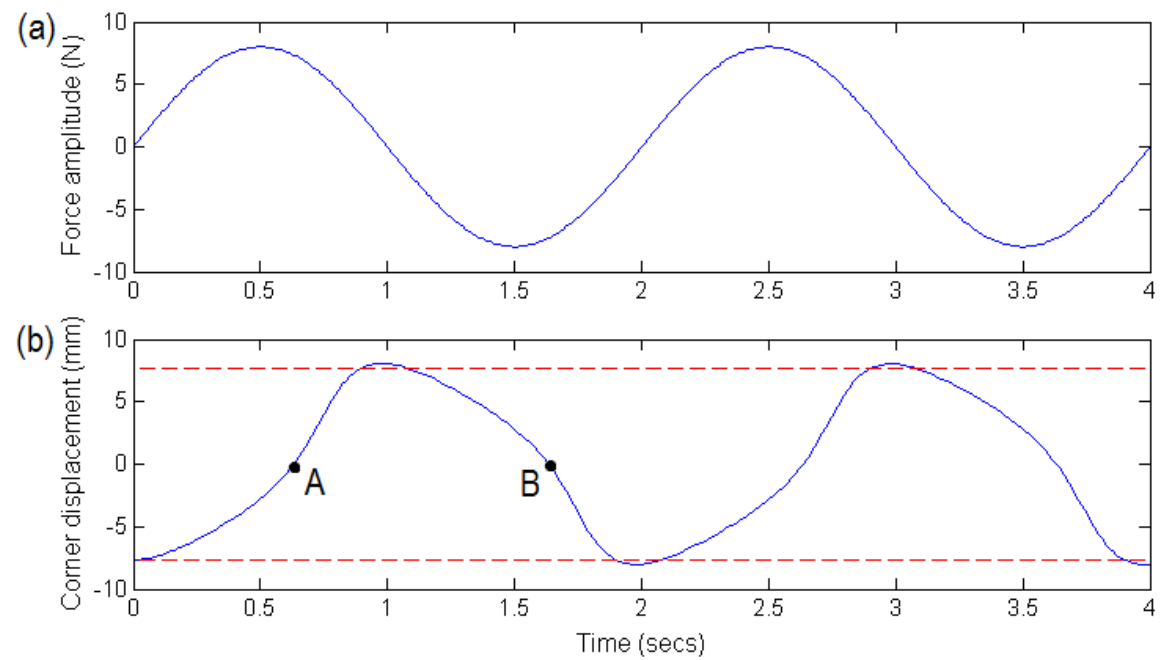

Figure 6. (a) Sinusoidal force excitation with a maximum amplitude of $8 \mathrm{~N}$, and (b) static stable shapes (dashed red lines) and dynamic actuation path (solid blue lines).

American Institute of Aeronautics and Astronautics 
The laminate is initially in the first stable state and has a negative corner displacement. The application of a positive force gradually reduces the laminate curvature and at approximately $0.7 \operatorname{secs}$ (see point A in Fig. 6b) a more marked change in the gradient of the displacement-time curve is observed. This corresponds to a transition to the second stable state with positive corner displacement. It is of interest to note that the laminate 'overshoots' the static stable position (dashed red line), inducing a small amount of additional curvature and therefore additional stress in the piezoelectric material. As the direction of applied force switches the laminate repeats the same cycle, transitioning from the second state to the first state at approximately 1.5secs (point B in Fig 6b), again overshooting the predicted stable position. This cycle then repeats uniformly with the sinusoidal force input.

We now investigate the optimality of the five ply $\left[0^{\mathrm{P}} / 0_{5} / 90_{5} / 90^{\mathrm{P}}\right]_{\mathrm{T}}$ global solution determined in Section III, with the additional consideration of the alternating force excitation. Figure 7 shows a measure of the harvestable energy (plotted on a logarithmic scale) for a range of different force amplitudes from $0.1 \mathrm{~N}$ to $10 \mathrm{~N}$. Three distinct regions (I $\rightarrow$ III, marked in Fig. 7) are noted; an inset plot with a linear scale is shown to highlight the magnitude of the change in energy generated in the three regions. Initally, in Region I, the energy harvested for low force amplitudes $(<6 \mathrm{~N})$ is found to be small but increases with additional force. This region can be investigated in more detail by considering a typical actuation cycle with a maximum force amplitude of $4 \mathrm{~N}$ (point A). Figure 8a shows a phase plot for this actuation cycle, plotting the applied force amplitude against the corner displacement of the laminate. The red dots indicate the two stable positions, while the blue line tracks the laminate displacement under the cyclic load. It is found that the energy harvesting device is oscillating about the first stable position at very low displacements, with no snap-through event to the second state. Figure $8 \mathrm{~b}$ confirms this behavior, illustrating the dynamic path of one corner of the laminate taken during the first six seconds of excitation.

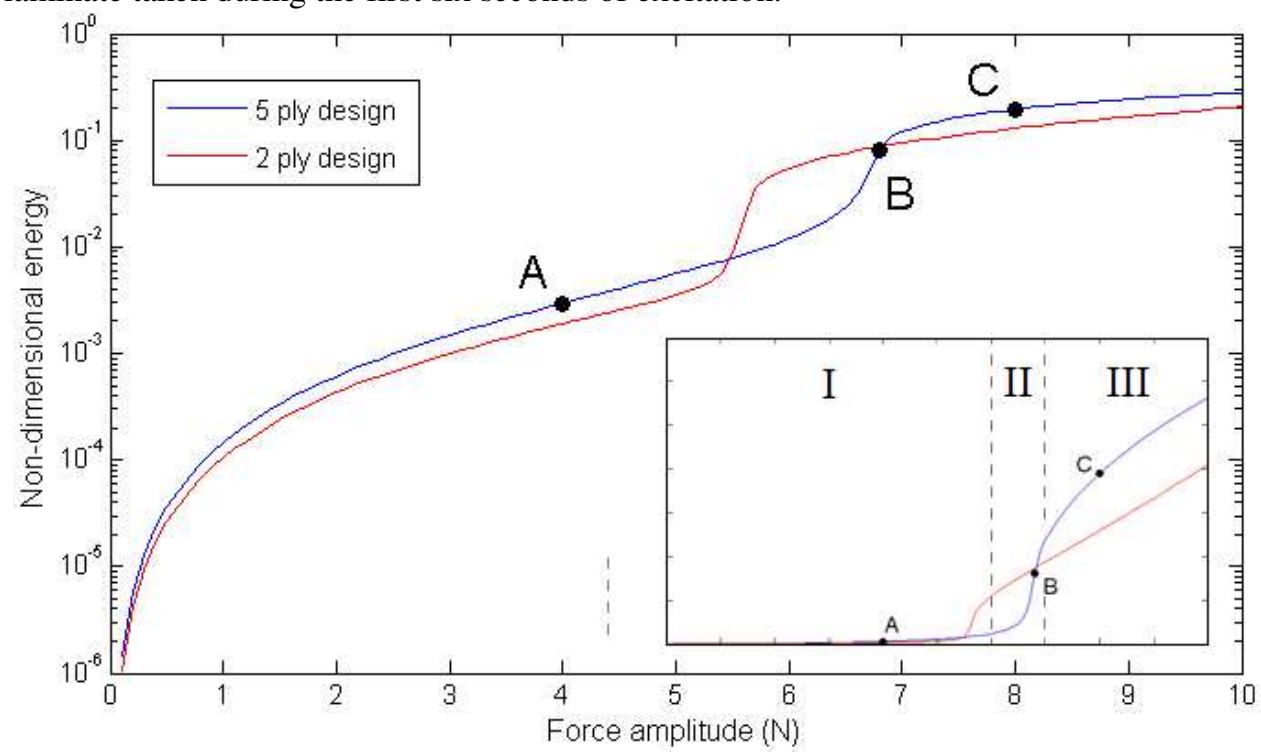

Figure 7. Harvestable energy levels (plotted on a logarithmic scale) for varying force amplitudes for a 5-ply laminate design corresponding to the optimal solutions of Section III, and a 2-ply design for comparison of actuation forces. Inset plot with a linear scale to highlight the magnitude change in energy generated.

In Region II between approximately 6 and $7 \mathrm{~N}$ in Fig. 7 there is a sharp increase in the harvested energy. Again, by selecting a point in this region $(6.8 \mathrm{~N}$ at point $\mathrm{B})$ we can investigate the dynamic behavior of this device. Figure $8 \mathrm{c}$ shows the associated phase plots. While the displacement of the device does not reach the second stable state, the corner displacement does crossover into the positive region, marking a transition into the second state region. Figure $8 \mathrm{~d}$ demonstrates that this region represents a finely balanced state of the system where snap-through has occurred but the direction change of the applied force at snap-through immediately forces the piezoelectric-laminate back towards the first state without ever reaching the second static stable position. However, this example demonstrates that, compared to Region I, higher levels of energy may be harvested at force levels which may not be sufficiently large to induce a full state change.

At higher force levels greater than $7 \mathrm{~N}$ (Region III) the electrical energy generated with force continues to increase but at a reducing rate (see Fig 7). If we consider point $\mathrm{C}$ at $8 \mathrm{~N}$ it can be observed that this region marks the point at which the device experiences a complete snap-through cycle encompassing both static stable states. Figure 8 e shows the phase plot for this actuation cycle and confirms that the device is oscillating between the two stable 
states, as indicated by the red dots. Figure $8 \mathrm{f}$ shows the dynamic path taken indicating a small overshoot beyond the static shapes and this behavior explains why the energy harvested continues to increase as the force is increased further. If the alternating force applied to the harvester continues to increase it will eventually result in straining of the piezoelectric material beyond the previously predicted states. The maximum strain constraint in the piezoelectric material, previously found to be inactive, is therefore likely to be an important consideration for optimization studies based on the dynamics of the system; especially since the failure strain of the piezoelectric material is much smaller that the carbon-fiber reinforced laminate ${ }^{18}$.
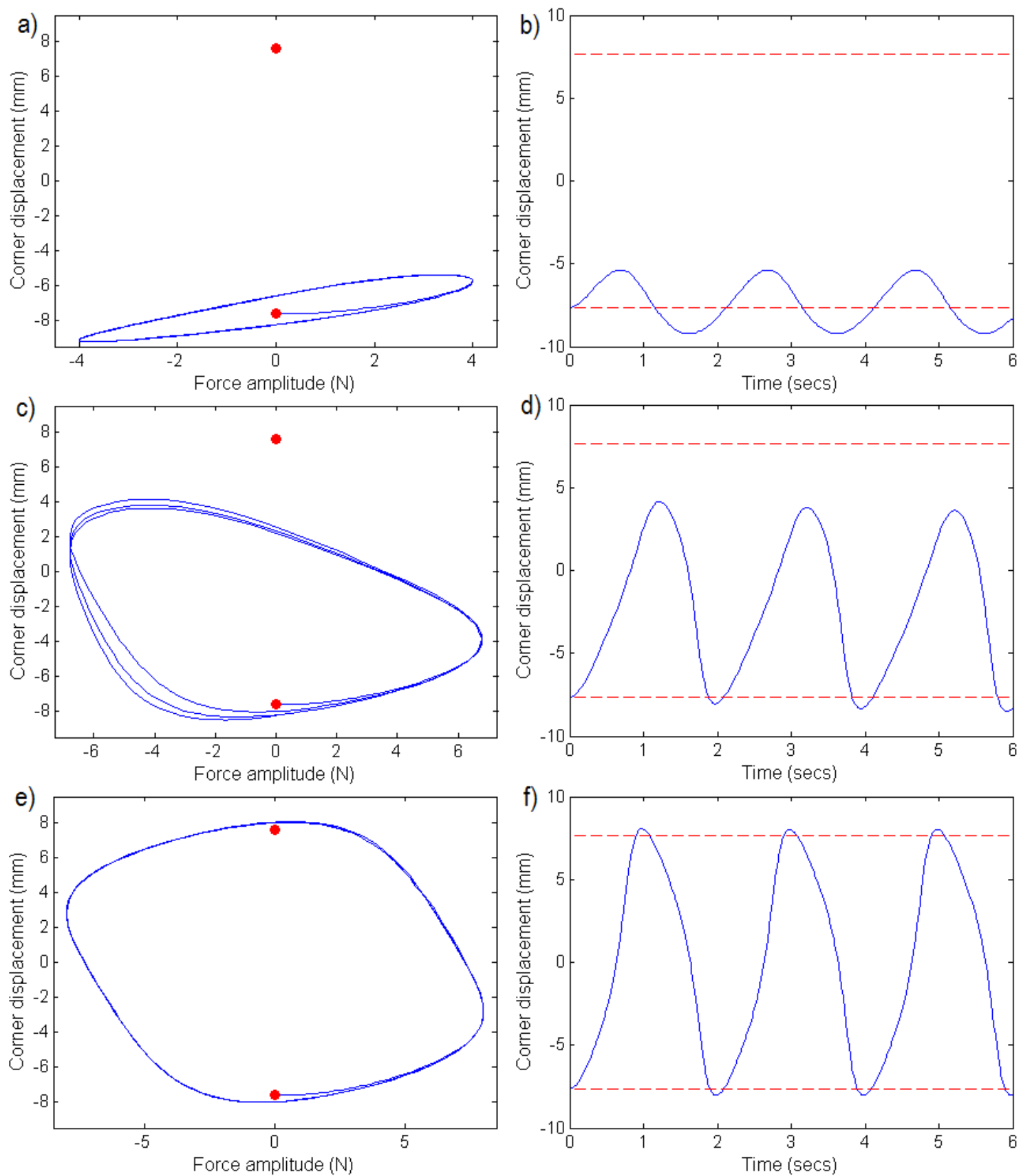

Figure 8. Dynamic response of a $\left[0^{\mathrm{P}} / 0_{5} / 90_{5} / 90^{\mathrm{P}}\right]_{\mathrm{T}}$ laminate with an osciallating force with maximum amplitude of (a-b) $4 \mathrm{~N}$, (c-d) $6.8 \mathrm{~N}$, and (e-f) $8 \mathrm{~N}$. Figures a, c, and e show the oscillating force displacement behavior around the two stable states (red dots). Figures $b$, $d$, and $f$ show the time varying pattern of corner displacement where stable positions are denoted by red dashed lines. 
For comparison, a second laminate design is also included in Fig. 7 to demonstrate an important consideration when using the optimal designs determined based purely on the statics of the system (Section III). The red line indicates the same energy generaton plot for varying force amplitudes for a thinner laminate design; $\left[0 \mathrm{P} / 0_{2} / 90_{2} / 90^{\mathrm{P}}\right]_{\mathrm{T}}$. With reference to Fig. $5 \mathrm{~b}$ this represents the optimal 2 ply design with $32.1 \%$ piezoelectric area and the same $0.2 \mathrm{~m}$ square edge length. We observe that when the dynamic response of the system is taken into account the thicker design is confirmed to be more optimal than the thinner design, producing more harvestable energy in the low force region where snap-through does not occur (Region I), and the high force region where bistability is fully exploited (Region III). However, the transition between these regions (Region II) occurs at a lower force for the thin laminate, resulting in the thinner laminate generating more electrical energy in this relatively narrow force range. In practical applications there is likely to be a constraint on the vibrational force amplitude and the high electrical energies of the thicker design may not be achieved. Constraints based on the ambient mechanical vibrations to be harvested are therefore an important consideration for optimization of the piezoelectric-laminate combination based on the dynamics of the system.

\section{Conclusion}

The optimization study presented in this paper has investigated the maximization of the electrical energy harvested of a bistable piezoelectric-laminate device due to the alternating stress excitation induced by repeated mechanical actuation. Constraints have been imposed to ensure bistability and to limit the strain in the piezoelectric material to below its failure strain. The results obtained in this study can aid in the determination of the optimum geometric configurations for energy harvesting and aid in the understanding of the underlying mechanism for high electrical energy generation in bistable piezoelectric-laminate based devices.

Through variation in ply orientations, laminate geometry and piezoelectric area it was found that cross-ply laminates $\left[0^{\mathrm{P}} / 0 / 90 / 90^{\mathrm{P}}\right]_{\mathrm{T}}$ offer the largest energy outputs since the laminate curvatures are maximized and aligned with the piezoelectric polarization axis. A local solution is also found $\left[0^{\mathrm{P}} / 90 / 0 / 90^{\mathrm{P}}\right]_{\mathrm{T}}$ with optimal piezoelectric alignment but reduced laminate curvature. The optimum ply thicknesses are found not to be on a constraint boundary, with thicker laminates that exhibit a lower curvature being more optimal than thinner high deflection designs. This pattern reverses as the geometry approaches the loss of bistability (see Fig. 1). Similarly, increased piezoelectric size with increased laminate thickness is found to be optimal, with the pattern reversing near the loss of bistability.

When considering the dynamic transition between stable states as the piezoelectric-laminate combination is exposed to an oscillating mechanical force, the optimum designs are found to be sensitive to the magnitude of the applied force. While the thicker laminates are confirmed to produce higher levels of energy when snap-through is fully induced, the force range in which snap-through occurs is more limited than for the less optimal, thinner designs. Furthermore, the strain in the piezoelectric material reaches higher levels than predicted based solely on the static states, suggesting the piezoelectric material failure constraint may indeed be active. In future optimization studies where the problem is constrained by dynamic considerations, tuning of the device for a specific vibration level is therefore essential.

\section{Acknowledgments}

We acknowledge the support of Engineering and Physical Sciences Research Council (grant number $\mathrm{EP} / \mathrm{J} 014389 / 1)$ for partially funding this work.

\section{References}

[1] Anton, S. R., and Sodano, H. A., "A review of power harvesting using piezoelectric materials (2003-2006)," Smart Materials and Structures, Vol. 16, No. 3, 2007, R1-R21.

[2] Mitcheson, P. D., Miao, P., Stark, B. H., Yeatman, E. M., Holmes, A. S., and Green, T. C., "MEMS electrostatic micropower generator for low frequency operation," Sensors and Actuators A: Physical, Vol. 115, No. 2-3, 2004, pp. 523529.

[3] Glynne-Jones, P., Tudor, M. J., Beeby, S. P., and White, N. M., “An electromagnetic, vibration-powered generator for intelligent sensor systems," Sensors and Actuators A: Physical, Vol. 110, No. 1-3, 2004, pp. 344-349.

[4] Arrieta, A. F., Hagedorn, P., Erturk, A., and Inman, D. J., "A piezoelectric bistable plate for nonlinear broadband energy harvesting," Applied Physics Letters, Vol. 97, No. 10, 2010, pp. 1-3.

[5] Priya, S., "Advances in energy harvesting using low profile piezoelectric transducers," Journal of Electroceramics, Vol. 19, No. 1, 2007, pp. 167-184.

[6] Erutrk, A., and Inman, D. J., "An experimentally validated bimorph cantilever model for piezoelectric energy harvesting from base excitations," Smart Materials and Structures, Vol. 18, No. 2, 2009, pp. 1-18. 
[7] Erturk, A., Hoffman, J., and Inman, D. J., "A piezomagnetoelastic structure for broadband vibration energy harvesting," Applied Physics Letters, Vol. 94, No. 25, 2009, pp. 1-3.

[8] Stanton, S. C., McGehee, C. C., and Mann, B. P., "Nonlinear dynamics for broadband energy harvesting: Investigation of a bistable piezoelectric inertial generator," Physica D: Nonlinear Phenomena, Vol. 239, No. 10, 2010, pp. 640-653.

[9] Hyer, M. W., "Calculations of the room-temperature shapes of unsymmetric laminates," Journal of Composite Materials, Vol. 15, (July) 1981, pp. 296-310.

[10] Dano, M. -L., and Hyer, M. W., "Thermally induced deformation behavior of unsymmetric laminates," International Journal of Solids and Structures, Vol. 35, No. 17, 1998, pp. 2101-2120.

[11] Tawfik, S. A., Dancila, D. S., and Armanios, E., "Planform effects upon the bistable response of cross-ply composite shells," Composites: Part A, Vol. 42, No. 7, 2011, pp. 825-833.

[12] Gude, M., Hufenbach, W., and Kirvel, C., "Piezoelectrically driven morphing structures based on bistable unsymmetric laminates," Composite Structures, Vol. 93, No. 2, 2011, pp. 377-382.

[13] Betts, D. N., Kim, H. A. and Bowen, C. R., "Optimization of stiffness characteristics for the design of bistable laminates," AIAA Journal, (Accepted).

[14] Betts, D. N., Kim, H. A. and Bowen, C. R., "Modeling and optimization of bistable composite laminates for piezoelectric actuation," Journal of Intelligent Material Systems and Structures, Vol. 22, No. 18, 2011, pp. 2181-2191.

[15] Priya, S., "Criterion for material selection in design of bulk piezoelectric energy harvesters," IEEE Transactions on Ultrasonics, Ferroelectrics and Frequency Control, Vol. 57, No. 12, 2010, pp. 2610-2612.

[16] Guillon, O., Thiebaud, F., and Perreux, D., "Tensile fracture of soft and harde PZT," International Journal of Fracture, Vol. 117, No. 3, 2002, pp. 235-246.

[17] Diaconu, C. G., Weaver, P. M., and Arrieta, A. F., "Dynamic analysis of bi-stable composite plates," Journal of Sound and Vibration, Vol. 322, No. 4-5, 2009, pp. 987-1004.

[18] Bowen, C. R., Dent, A. C., Nelson, L. J., Stevens, R., Cain, M. G., and Stewart, M., "Failure and volume fraction dependent mechanical properties of composite sensors and actuators," Proceedings of the IMechE, Part C: Journal of Mechanical Engineering Science, Vol. 220, No. 11, 2006, pp. 1655-1663. 